\title{
Targeted intraoperative radiotherapy in oncology by Keshtgar et al.
}

\author{
Cory Donovan • Charlotte Dai Kubicky
}

Received: 25 August 2014 / Accepted: 3 September 2014 / Published online: 21 September 2014

(C) Springer-Verlag Berlin Heidelberg 2014

Surgical treatment of early stage breast cancer has shifted over the last 50 years from a radical to a less invasive approach. Postoperative radiation is essential to breast conservation. External beam radiation to the whole breast followed by a boost to the operative bed has been the standard of care. The prolonged time commitment from patients and normal tissue toxicities has spurred the development of alternative radiation techniques. Accelerated partial breast irradiation (APBI) was developed to shorten the treatment course and to minimize doses to normal tissue. The Zeiss Intrabeam is a mobile, low energy X-ray device for APBI. Following surgical excision of a breast tumor, accelerated electrons strike a gold plate, emitting low-energy $\mathrm{x}$-rays through a spherical applicator directly into the tumor bed. A number of clinical trials have evaluated the efficacy of Targeted Intraoperative Radiotherapy (TARGIT) in breast cancer, and it is under investigation for the treatment of other malignancies.

Targeted Intraoperative Radiotherapy in Oncology was written by a multidisciplinary panel of experts who have been involved in the development of TARGIT for many years. They have created an illustrated guide to intraoperative radiotherapy, using TARGIT, for clinicians from a variety of disciplines. They begin with the rationale for the development of this technology and go on to review results from the TARGITA trial, a phase III randomized controlled non-inferiority trial. The chapter also discusses other trials employing TARGIT and future research directions. In the first few chapters, they describe the technology in accessible language for clinicians who are less familiar with radiation physics and with the Intrabeam device in particular. The subsequent chapters veer to the more technical aspects of this technology. Sequential screen captures illustrate the step by step safety checks and the

C. Donovan · C. D. Kubicky $(\bowtie)$

Oregon Health Science University, Portland, Oregon, USA

e-mail: charlottedai@gmail.com practical use of the components, including a trouble-shooting flow chart.

While earlier chapters are written to appeal to radiation oncologists and physicists, subsequent chapters address the interests of other specialties. The illustrations in the surgical considerations chapter were particularly useful, creating a clear visual image of the device in a prepared surgical field. There is a chapter addressing specific postoperative findings on imaging for radiologists, as well as discussions on quality of life, cosmetics, and persistent pain following TARGIT. On occasion, certain chapters spend too much time exhaustively describing their methodology, but the conclusions are informative. Several additional chapters address novel uses for the Intrabeam device in soft tissue sarcomas, brain tumors, endometrial cancer, and rectal cancer recurrence. The authors then present a number of case studies that pose therapeutic dilemmas and where TARGIT can be used. Some of the most thought-provoking chapters address intraoperative radiotherapy in developing countries and the health economics of TARGIT.

On the whole, this book is easy to read, focused, and accessible to a broad audience. There are chapters of specific interest to radiologists, oncologists, surgeons, radiation oncologists, and physicists. The illustrations and detailed practical information are strengths of this publication. It is clear that the authors believe strongly in the key role of TARGIT in the treatment of breast cancer. However, because of their strong belief, the authors may be biased in the clinical efficacy of TARGIT. To fully evaluate the role of TARGIT, we await the long-term clinical outcome data from the phase III randomized TARGIT-A trial, that randomized patients to risk-adapted targeted intraoperative radiotherapy versus whole breast radiotherapy.

\section{Conflict of interest None.}

Ethical Standards Statement The manuscript does not contain clinical studies or patient data. 